\title{
Aprendizaje por problemas: una experiencia en el grado de Ingeniería en Tecnologías Industriales \\ Problem Based Learning: Experience in the Industrial Technologies Engineering Bachelor Degree
}

\author{
Sophie Gorgemans ${ }^{1}$, José Tomás Alcalá Nalvaiz ${ }^{2}, \mathrm{M}^{\mathrm{a}}$ Inmaculada Gómez-Ibañéz ${ }^{3}$ \\ ${ }^{1}$ sgorge@unizar.es \\ Dirección y Organización de Empresas \\ Universidad de Zaragoza \\ Zaragoza, Spain \\ 2 jtalacla@unizar.es \\ Métodos Estadísticos \\ Universidad de Zaragoza \\ Zaragoza, Spain \\ 3 igomez@unizar.es \\ Matemática Aplicada \\ Universidad de Zaragoza \\ Zaragoza, Spain
}

\begin{abstract}
Resumen- La sociedad pide un perfil de egresados que sean capaces de tener agilidad en la resolución de problemas así como capacidad para trabajar de forma cooperativa y dirigir equipos. Así surge la oportunidad para la Universidad de desarrollar metodologías activas de aprendizaje desde los primeros cursos de grado. Para ello, en esta experiencia, se han coordinado los profesores de distintas materias para diseñar problemas con un proceso de resolución basado en el trabajo en equipo. Los alumnos que han participado en la misma obtienen una doble satisfacción, tanto a nivel cuantitativo por verse incrementada su calificación, como a nivel cualitativo por afianzar algunas de las competencias transversales. Los resultados obtenidos en un cuestionario ad-hoc de evaluación revelan que se trata de una actividad prometedora y que debería extenderse a más asignaturas y otros contextos.
\end{abstract}

Palabras clave Aprendizaje basado en problemas, Trabajo en equipo, Interdisciplinridad, Grados en Ingeniería

\begin{abstract}
Our society demands profile where the graduates are able to have agility in solving problems as well as in working cooperatively and managing teams. This is a great opportunity for the University to develop active learning methodologies from the first degree courses. In this teaching experience, some teachers of different subjects have designed a set of problems which resolution process was based on teamwork. The participating students obtained a double satisfaction, a quantitative one increasing its qualifications, and a qualitative one strengthening some of the transversal key competences. The results obtained in an ad-hoc evaluation questionnaire revealed that this is a promising activity and should be extended to more subjects and other contexts.
\end{abstract}

Keywords Problem Based Learning, Teamwork, Interdisciplinary, Engineer's Bachelor Degrees

\section{INTRODUCCIÓN}

El ejercicio de la profesión de ingeniero requiere que los profesionales puedan resolver problemas complejos en situaciones de incertidumbre o incluso con informaciones incompletas (Mills y Treagust, 2003). Además, el desempeño del trabajo se inscribe generalmente en un entorno cooperativo que se apoya en competencias comunicativas. De hecho, las expectativas de los empresarios del sector industrial respecto a los nuevos egresados de las ingenierías están centradas en aptitudes como la iniciativa, el liderazgo, la comunicación, el trabajo y la dirección de equipos, la capacidad de adaptación, así como las facultades de negociación y de toma de decisiones.

La preocupación de la Universidad por mejorar la empleabilidad de sus egresados pasa por una mayor adecuación del perfil de los ingenieros a la demanda de la sociedad y adecuar los métodos de enseñanza-aprendizaje a la adquisición de las competencias que podrán hacer valer en el mercado laboral. Así, a los métodos tradicionales de enseñanza se suman metodologías activas como el aprendizaje basado en problemas y el trabajo en equipo, principales pilares de la experiencia llevada a cabo en este estudio.

El aprendizaje basado en problemas (ABP) es una herramienta pedagógica que da fuerza a los estudiantes para conducir una investigación, integrar teoría y práctica y aplicar los conocimientos y las habilidades para desarrollar una solución viable a un problema determinado (Savery, 2006). Esta metodología, inicialmente utilizada en los estudios de medicina y otras ciencias de la salud, se ha extendido durante los últimos años a distintos niveles de enseñanza como los programas de MBA (Stinson y Milter, 1996), enseñanza superior (Bridges y Hallinger, 1996); y a titulaciones de diversas áreas, en particular a las ingenierías (Perrenet et al., 2000; Heitmann, 1996; Woods, 1994; Woods et al., 2000; Yadav et al. 2010 y 2011). Esta lista, lejos de ser exhaustiva, pretende simplemente ser ilustrativa de los múltiples contextos en los cuales el ABP puede ser utilizado. En los grados de Ingeniería es particularmente útil para confrontar los estudiantes a numerosas situaciones en las que se verán reflejados y entrelazados los contenidos de las materias básicas (instrumentales) y de las materias específicas así como con casos reales que van a encontrar en el ejercicio de su profesión (máquinas, herramientas y/o procesos industriales en empresas).

Con el ABP, además de la reflexión individual que hace el estudiante acerca del problema, la propuesta de solución se obtiene habiendo ampliado esta primera reflexión en el grupo formado por varios estudiantes. La competencia relativa al trabajo en equipo está incorporada en los planes de estudios de 
todas las ingenierías y el desarrollo de la misma deja evidencias de tres tipos: individuales, colectivas y de resultados (Fidalgo et al., 2015). El dominio del estudiante sobre el tema a tratar y su nivel de participación en el grupo son dos aspectos esenciales en esta técnica de aprendizaje colaborativo. El perfil del líder del grupo puede llegar a condicionar el resultado que obtenga el mismo. Por ello, es tan importante evaluar los resultados alcanzados como medir las percepciones que tengan los participantes acerca del funcionamiento del grupo.

Entre otras ventajas el ABP permite ampliar los conocimientos de forma significativa, puesto que el estudiante comprende bien las variables que afectan al problema, así como las diferentes alternativas y soluciones al mismo.

Asimismo, al ser los protagonistas en el proceso de desarrollo y búsqueda de solución, es decir, al realizar la tarea de forma activa, los estudiantes retienen mejor el conocimiento adquirido, y desarrollan nuevas competencias transversales imprescindibles para su futura profesión: aprenden a comunicar sus ideas eficazmente, analizan los problemas de un modo crítico, desarrollan su capacidad para tomar decisiones acertadas, se vuelven más curiosos y su interés por aprender aumenta.

La mayoría de las veces el problema se diseña enfocándolo a una única asignatura, pero cuando se coordina y adquiere un diseño interdisciplinar, los beneficios son mayores (Diaz et al., 2015). El objetivo de este trabajo consiste en integrar el sistema de aprendizaje en un amplio rango de disciplinas, diseñando problemas multidisciplinares de tal manera que, en su resolución, los estudiantes necesiten recurrir a conocimientos básicos (matemáticos) así como a los conocimientos específicos desarrollados en la materia en la que esta metodología activa se utiliza.

En el siguiente apartado se definen los objetivos, el contexto y el público que ha podido participar en esta experiencia innovadora.

\section{CONTEXTO}

La experiencia reflejada en este estudio es parte de un proyecto en el que se encuentran involucrados siete profesores de áreas de conocimiento muy distintas, pero cuyo nexo común es impartir docencia en el mismo centro universitario, implicando a múltiples asignaturas de primer y segundo curso en las titulaciones de Ingeniería Técnica Industrial (4 asignaturas) e Ingeniería Eléctrica (IE) en la Escuela de Ingeniería de la Universidad de Zaragoza.

Las asignaturas se han coordinado por pares con el fin de facilitar el diseño y seguimiento de los casos. En todos los casos la materia de Matemáticas, impartidas en el primer curso, formaba parte del binomio. Esta elección se justifica por el convencimiento que teníamos que muchos de los contenidos instrumentales de la materia se deberían asimilar mejor cuando se ponen en referencia con los contenidos del resto de asignaturas participantes en la experiencia (Fundamentos de Administración de Empresas, Mecánica de Fluidos y Motores Eléctricos). La mayoría de estas asignaturas están estructuradas en torno a cuatro tipos de actividades docentes: clases magistrales, prácticas, actividades de evaluación y trabajo dirigido y, es en esta última actividad que se ha estructurado y desarrollado el ABP.
La actividad se ha aplicado a un primer curso de grado, donde el encaje entre asignaturas instrumentales y básicas resulta más sencillo. Su aplicabilidad a otros contextos es total, si bien no hay que perder de vista la coordinación de contenidos entre dos (o más) asignaturas y también, la secuenciación y proximidad temporal entre las asignaturas involucradas en el ABP multidisciplinar.

Para que fueran un éxito y generaran interés en los estudiantes, los problemas se diseñaron partiendo de un caso real (de empresa y de máquinas que los estudiantes van a encontrar en el ejercicio de su desempeño profesional: un motor eléctrico y una bomba).

En el diseño de los problemas se han tenido en cuenta las siguientes características:

- Los estudiantes tenían la responsabilidad de su propio aprendizaje

- El problema definido debe dejar suficiente libertad para encontrar y descifrar su complejidad.

- Como la colaboración entre los miembros del grupo es esencial, el tutor preguntaría a los estudiantes indistintamente para asegurarse que la información ha sido compartida por todos.

- Lo que los estudiantes aprenden durante el proceso debe verse aplicado en el problema con un nuevo análisis y resolución.

- Un análisis cerrado de lo que se ha aprendido del trabajo con el problema y la discusión de los conceptos y principios que se han estudiado son esenciales.

- La resolución del problema implica un trabajo personal de cada estudiante así como una relación entre estudiantes y entre grupo de estudiantes y profesor-tutor.

- La evaluación de los estudiantes debe medir el progreso de los mismos hacia los fines del aprendizaje con problemas: adquisición de conocimientos y en la realización del proceso. Tienen que poder estructurar lo que saben y lo que han aprendido.

Para el diseño de los problemas los profesores participantes en la experiencia compartieron, en primer lugar, información sobre los contenidos para enfocar así el problema hacia campos efectivamente tratados en las materias. Posteriormente se organizaron los materiales y se ideó el procesamiento de la información que los estudiantes tendrían que realizar para verificar así si todos los elementos de información estuvieran en el enunciado o, al menos, su ausencia pudiera ser detectada y rápidamente cubierta por búsquedas sencillas a realizar por los propios estudiantes.

El papel del tutor ha sido doble ya que ha facilitado el proceso de aprendizaje como gestor del proceso, y ha actuado como un entrenador de las habilidades metacognitivas de los estudiantes animándoles a alcanzar un nivel más profundo en el entendimiento del problema y asegurando que todos los estudiantes, en cada grupo, estuvieran activamente involucrados en el proceso de análisis y resolución propuesto (Barrows, 1988). 
Los estudiantes formaron los grupos con total libertad, juntándose por motivaciones diversas que, a posteriori, se analizaron en el cuestionario de evaluación. Para valorar el resultado obtenido cada uno de los tutores tomó en consideración el desarrollo escrito $y$, por otro lado, las habilidades comunicativas a la hora de explicar la resolución así como el proceso de toma de decisiones seguido. De la primera parte, los estudiantes de un mismo grupo lograron la misma nota mientras que, de la exposición oral de ideas, obtuvieron una valoración cualitativa recibiendo un feed-back directo del tutor al acabar la sesión.

En la siguiente sección, se describe con mayor detalle las actividades llevadas a cabo por los profesores que participan en esta experiencia y el desarrollo del proceso en sí hasta su etapa final de evaluación de resultados para la que se ha diseñado un cuestionario ad hoc.

\section{DESCRIPCIÓN}

En una primera etapa, los profesores que forman el grupo de innovación docente han centrado su esfuerzo en elaborar una serie de problemas cuya resolución requería usar tanto los conocimientos específicos de las materias como los conocimientos instrumentales de matemáticas desarrollados en dos asignaturas del primer curso.

Se ha trabajado por parejas de asignaturas en el ámbito de una misma titulación para garantizar la continuidad del alumnado entre una y otra materia. En todos los casos se ha seguido el mismo plan de actuación para el desarrollo del caso en el aula. Así, se clarificó, en primer lugar, que se trataba de una actividad formativa que pretendía abrirles los ojos sobre la interacción de materias en la aplicación de casos reales de ingeniería.

La temática de los problemas desarrollados ha sido amplia, por ejemplo:

- métodos numéricos de resolución de ecuaciones diferenciales ordinarias con problemas de máximo flujo

- $\quad$ sistemas de ecuaciones y motores trifásicos

- aplicación del álgebra lineal a modelos de producción (modelo de Leontief)

- aplicación del cálculo matricial a la aproximación a las ventas en una empresa

En el área de Matemáticas para la ingeniería se imparte generalmente en los primeros cursos el cálculo diferencial e integral, el álgebra lineal y las ecuaciones diferenciales, entre otros. El proceso de enseñanza-aprendizaje en estas asignaturas no resulta sencillo ya que frecuentemente se suelen lograr competencias algorítmicas y algebraicas aunque habitualmente los contenidos matemáticos quedan desvinculados de la problemática que se aborda en otras asignaturas de las diferentes ingenierías. Ante esta situación surge la idea de reflexionar sobre el papel que tiene el docente como un mediador de una interacción educativa capaz de propiciar en el estudiante el desarrollo de las habilidades y actitudes que les permitan utilizar los conocimientos matemáticos para el análisis requerido en las diferentes disciplinas de las ingenierías y la necesidad de interactuar desde los primeros cursos con otras asignaturas.

En segundo lugar, se trasladó la aplicación al aula en diversas etapas: 1) una sesión de presentación del proyecto por los dos docentes cuyas materias se entrelazan; 2) una sesión de concreción del trabajo con todos los estudiantes voluntarios; 3) realización del trabajo a resolver en equipo - 3 semanas; 4) una sesión de evaluación del trabajo; y para finalizar 5) la evaluación de la metodología.

Los estudiantes formaron los grupos de trabajo a su libre albedrío respetando el número de integrantes impuesto por el profesor (cinco miembros en todos los casos). En la tutoría inicial de concreción del ABP, los profesores explicaron a sus estudiantes las características principales de esta metodología y repartieron los enunciados. Cada tutor, en la segunda sesión, les hizo una breve exposición sobre el trabajo en equipo en la que se insistió sobre los roles que podían asumir en el grupo y las herramientas que tenían a su alcance para gestionar las reuniones necesarias para compartir la información y alcanzar una solución, indicando a su vez, la necesidad de elaborar reglas de comportamiento para el grupo, de procedimientos a establecer para garantizar su correcto funcionamiento - como por ejemplo la redacción de actas de las reuniones celebradas.

A lo largo de las siguientes semanas, los estudiantes acudieron a tutorías para resolver dudas de planteamiento o de resolución. En el momento de la sesión de evaluación se debatieron entre estudiantes y profesor la resolución del problema indagando en los procesos de toma de decisión adoptados.

Con el objeto de poder medir el impacto de la innovación, se ha diseñado un cuestionario (en fase piloto) que es entregado a los alumnos tras la tutoría final y que rellenan voluntariamente en ese momento. (por tanto sin grupo de control). Cuando el procedimiento esté totalmente asentado, por cada ABP y alumno participante, se tendrán dos encuestas que nos permitirán medir tanto el nivel de satisfacción como la evolución del alumno que sigue esta metodología.

A continuación se presenta un breve resumen de los resultados académicos asociados a los problemas planteados en este curso académico. Se describen los resultados de los cuestionarios completados (estamos a la espera que nos lleguen más cuestionarios completados a lo largo de esta semana y podamos mejorar el análisis posterior y conclusiones). Finalmente, se valoran la validez y fiabilidad del cuestionario, como instrumento de medida, para plantear posibles mejoras en su versión final.

\section{RESUltados}

En la parte de la investigación cuyos resultados se presentan, es de destacar una gran participación en la asignatura de primer semestre. En esta asignatura, participaron un total de 12 grupos, cada uno de 5 alumnos. Todos los grupos presentaron sus materiales, aunque algunos de ellos no estaban completos. En la asignatura en la que el ABP continuaba la experiencia sólo pudo llevarse en dos subgrupos de prácticas al tener el profesor en este cuatrimestre responsabilidad docente sobre dos de los cuatro grupos de prácticas, es decir, a unos 30 estudiantes, con un total de 6 grupos. El nivel de participación alcanzado es actualmente del $33,3 \%$ en ese caso.

Desde un punto de vista académico en la primera asignatura, supuso en todos los casos un aumento de la calificación final. A falta de elementos de juicio individualizados, se optó por valoración única grupal. Sobre un máximo de 0,5 puntos en la 
nota final, más de la mitad de los grupos alcanzaron una puntuación mayor o igual a 0,3.

En la segunda asignatura Fundamentos de Administración de Empresas, el problema se ofertó a los estudiantes unas tres semanas antes de que se acabara el cuatrimestre lo que condicionó mucho el volumen de respuestas recibidas porque coincidía con una época de múltiples entregas y del último parcial en la asignatura de Física II. Los estudiantes recibieron varios avisos tanto desde su profesor de teoría en el aula como por parte del profesor de prácticas en el aula y mediante correo electrónico para animarles a participar. Además de estos inconvenientes temporales se sumó otro aspecto importante: la resolución del caso no suponía ningún incremento de la nota ya que la guía docente no daba ninguna posibilidad a que este ejercicio pudiera ser considerado.

\section{A. Valoración de la actividad formativa}

El cuestionario se ha estructurado en 4 partes (Figura 1). La primera es una parte identificativa de las características del estudiante (edad, sexo, número de veces matriculado en la asignatura, motivaciones y papel asumido en el grupo), la segunda se centra en las dificultades, la tercera en los puntos de interés y la última sobre el nivel de satisfacción alcanzado a través de la experiencia. En cada una de las partes se pide valorar cuatro aspectos específicos utilizando una escala de Likert de 5 puntos (graduada de muy poco a mucho).

El cuestionario se diseña de forma que se pueda completar sin supervisión del profesor y que sea rápido de contestar (la duración aproximada para completarlo es de 4 minutos). La existencia de otros cuestionarios orientados a evaluar una asignatura completa como el modelo de encuesta SEEQ (Students' Evaluation of Educational Quality) o a evaluar los enfoques de aprendizaje como el R-SPQ-2F no se han considerado totalmente adecuados a nuestros objetivos iniciales (Biggs, Kember y Leung, 2001). En cualquier caso, esta versión piloto deberá ser revisada a la luz de los resultados y los nuevos objetivos que se vayan incorporando en el futuro.

Los cuestionarios de evaluación de la actividad se rellenaron por parte de 57 alumnos en la primera asignatura y 2 en la segunda [estamos pendientes de recibir más respuestas esta semana que los estudiantes completaran en la sala de examen].

Respecto a los resultados de evaluación de la actividad cabe destacar un alto grado de satisfacción generalizado por parte de los alumnos que han participado. Sin duda existía un esfuerzo añadido por adquirir algunos contenidos por adelantado de forma autónoma, consecuencia del carácter interdisciplinar de la actividad. En general los alumnos percibieron esto como un reto que asumieron con bastante motivación.

Para el 75,4\% de ellos se trataba de su primera actividad de aprendizaje con contenidos interdisciplinares. Es un valor muy alto y que identifica claramente un tipo de actividad novedosa para los alumnos de primer curso.

La formación de grupos se realizó en su mayoría por afinidad-amistad o por coincidencia previa en otras actividades grupales (algo más del $80 \%$ de casos); sin embargo el azar o amistad en etapas anteriores son opciones menores.

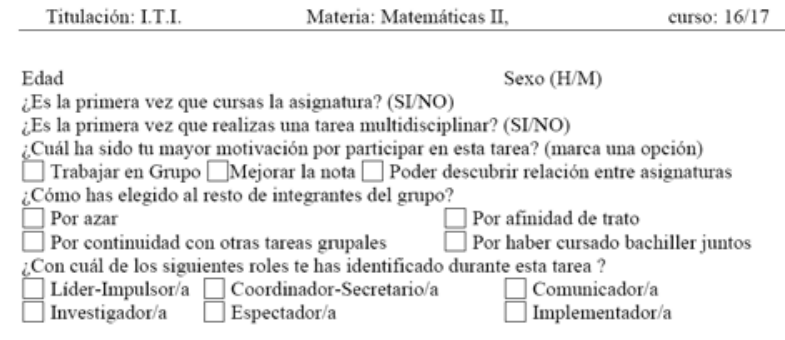

Valora el dificultad que te ha supuesto los aspectos que se indican. En todos los casos la puntunción va de 1 (muy poco) a 5 (muchos)

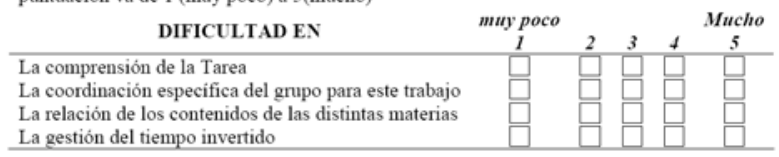

Valora el grado de interés que han suscitado los siguientes aspectos. En todos los casos la puntuación va de 1 (muy poco) a 5 (mucho)

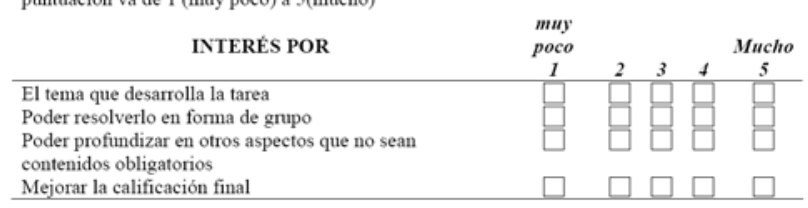

Mejorar la calificación final

Valora la satisfacción global

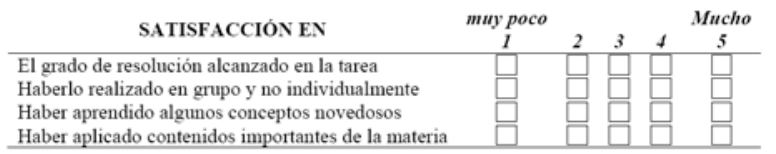

Haber aplicado contenidos importantes de la materia

Comentarios que desees realizar:

Figura 1 Encuesta facilitada

Entre las motivaciones que han determinado la participación en la actividad destaca la de mejorar la nota, quedando en un segundo nivel igualado las otras dos opciones que se ofrecían tales como el trabajo en grupo o la posibilidad de relacionar asignaturas (Figura 2). Estas dos últimas motivaciones están más alineadas con las motivaciones y estrategias de un enfoque profundo frente a un enfoque superficial del aprendizaje.

\section{MOTIVACIÓN}

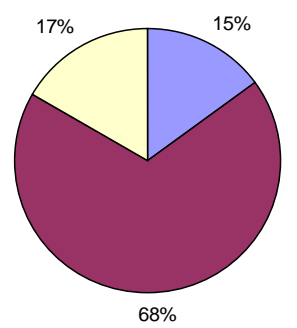

$\square$ trabajo-grupo

- mejora-nota

$\square$ relacionar-asignaturas

Figura 2 Frecuencia en la motivación al participar

Los alumnos se han identificado mayormente con los roles de investigador y secretario (entre los dos, el 60\% de los alumnos). Sin embargo, las figuras del líder, del comunicador o del implementador (programador de rutinas) han recibido menos atención (Figura 3). Estos roles son más novedosos y tal vez requieren una formación más específica por parte del profesorado cuando son explicados dentro de una actividad de trabajo por grupos. Ha sido de agrado ver que ningún 
participante se ha autocalificado como espectador, todos entendieron que debían asumir un rol activo.

\section{Rol en el Grupo}

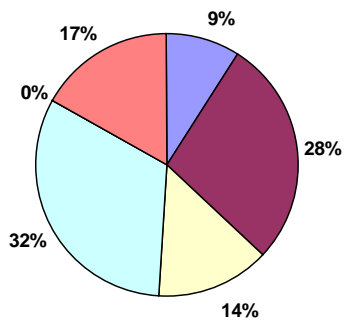

Figura 3 Frecuencia del Rol asumido en el grupo

Las tres dimensiones valoradas en la encuesta eran: interés, dificultad y satisfacción (Figura 4).

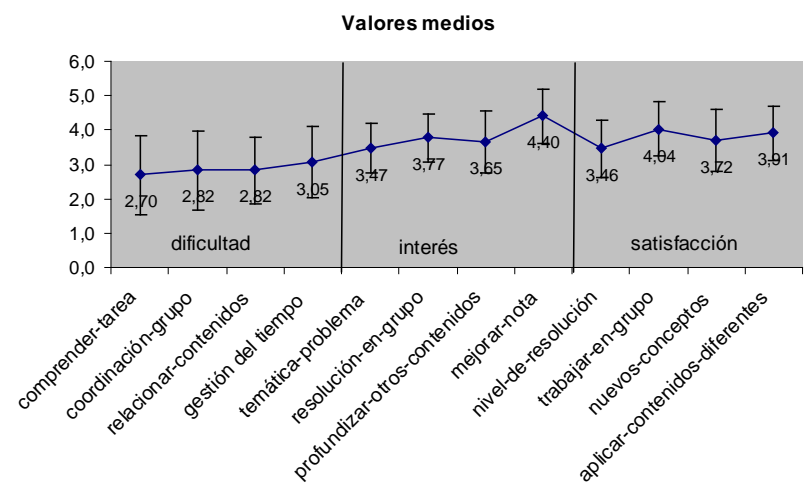

Figura 4 Valoración media de los ítems del cuestionario

Respecto al interés, hay una motivación especialmente bien considerada, la mejora de la calificación final. El resto de motivaciones están en niveles próximos a 4, salvo la temática de los problemas que queda ligeramente por debajo de 3,5.

Respecto a la dificultad, en la figura 4 se puede apreciar valores medios en torno a 3 indicando una dificultad media.

Finalmente respecto a la satisfacción, en la figura 4 podemos ver valores medios próximos o superiores al 4, salvo en el grado de resolución alcanzado donde el nivel de satisfacción medio es de 3,46.

\section{B. Validez y fiabilidad del instrumento de medida}

El cuestionario como instrumento de medida presenta un alto valor de fiabilidad con un alfa de Cronbach global de 0,77. Por dimensiones (grupos de preguntas) el valor de este estadístico baja en todos los casos (Tabla 1).

Tabla 1 Índices de fiabilidad globales y por grupos de items

$$
\begin{array}{c|c|c|}
\hline \text { N. } & \text { ALFA } & \text { ALFA } \\
\hline \text { ÍTEMS } & & \text { TIPIFICADO } \\
\hline
\end{array}
$$

\begin{tabular}{l|ccc}
\hline DIFICULTAD & 4 &, 692 &, 695 \\
INTERÉS & 4 &, 491 &, 500 \\
SATISFACCIÓN & 4 &, 649 &, 643 \\
TODAS & 12 &, 763 &, 770
\end{tabular}

El análisis factorial exploratorio muestra un valor del estadístico KMO medio-alto, de 0,624 y un número de 4 factores subyacentes. Estos factores pueden resumirse en:

- factor 1: interés/satisfacción por aplicar y adquirir nuevos contenidos del resto de asignaturas.

- factor 2: de interés/satisfacción ante nueva forma de trabajo (el trabajo en grupo).

- factor 3: dificultad en el trabajo en grupo

- factor 4: eficiencia-eficacia de tiempo invertido y resultados alcanzados

Las dificultades para obtener encuestas tras la segunda asignatura involucrada en el $\mathrm{ABP}$ ha limitado tanto la posibilidad de medir la evolución de las valoraciones y también la fiabilidad del cuestionario mediante técnicas de test-retest.

Tras esta primera versión, parece adecuado plantearse un cuestionario reagrupando las preguntas en torno al trabajo en grupo, el problema ABP que se aborde, la labor tutorial y de coordinación del profesorado; ya que este aspecto quedaba sin cubrir en este primer cuestionario. Esta reagrupación permitiría focalizar más las dimensiones subyacentes y disponer de una herramienta de control y mejora para el profesorado involucrado en esta actividad.

\section{CONCLUSIONES}

Se trata de un proyecto que usa metodología $A B P$ por grupos, destacando su carácter interdisciplinar. Permite un uso coordinado de metodología y materiales entre asignaturas, y que se espera ser extensible a un número mayor de asignaturas de los primeros cursos.

Los resultados tanto académicos como los asociados a la valoración son prometedores. El instrumento de valoración de la actividad se revela como un instrumento adecuado y fiable. Aun así, puede ser necesaria alguna revisión y mejora. Para futuras implementaciones se debería arbitrar mecanismos de evaluación más personalizada a cada individuo que reflejara tanto los niveles de resolución como la participación y el proceso de trabajo realizado. Se podría introducir mecanismos de evaluación por iguales en cada uno de los grupos. Sería conveniente considerar una evaluación de lo que los estudiantes han aprendido con el problema y discutir en profundidad los conceptos y principios que han estudiado.

Los resultados obtenidos respecto a la participación demuestran que resulta fundamental dar opción a que los estudiantes sean evaluados y que el resultado de dicha evaluación se sume al resto de notas que pueden obtener con las demás actividades planificadas. Parece también esencial evitar la desconexión entre las materias por lo que se aconsejaría la presencia de los dos profesores responsables en el momento de la presentación de cada uno de los problemas aunque éstos correspondan a materias distintas y, a lo mejor, a cuatrimestres de impartición diferentes. Podría resultar de interés para la medición del resultado añadir una pregunta en el cuestionario sobre la continuidad percibida entre los dos problemas.

Sin lugar a dudas quedan aspectos por profundizar o desarrollar como el control sobre la adquisición de las 
competencias en el trabajo en grupo o la evaluación de los roles asumidos.

\section{AGRADECIMIENTOS}

Esta investigación ha sido financiada por la universidad de Zaragoza a través de los proyectos de la convocatoria de innovación docente PIIDUZ 2015-16 (15-129) y PIIDUZ 2016-17 (16-021).

\section{REFERENCIAS}

Barrows, H.S. (1988). The Tutorial Process, Springfield, Illinois: Southern Illinois University School of Medicine.

Biggs J.B., Kember D. y Leung D.Y.P. (2001) The Revised Two Factor Study Process Questionnaire: R-SPQ-2F. British Journal of Educational Psychology, 71, 133-149.

Bridges E.M. y Hallinger, P. (1996). Problem-based learning in leadership education. New directions For Teaching and Learning, 1996(68), 53-61.

Diaz-Garrido E., Martín-Peña M.L. y López-Sánchez J.M. (2015). E-learning y coordinación interdisciplinar: Una experiencia con aprendizaje cooperativo, CINAIC 2015. Madrid.

Fidalgo-Blanco A., Sein-Echaluce M.L., García-Peñalvo F. y Conde M.A. (2015). Using Learning Analytics to improve teamwork assessment. Computers in Human Behavior, 47, 149-56.

Heitmann G. (1996). Project-oriented study and Projectorganized curricula: A brief review of intentions and solutions. European Journal of Engineering Education, 21(2), 121-31.
Mills, J. E. y Treagust, D. F. (2003). Engineering education Is problem-based or project-based learning the answer? Australasian Journal of Engineering Education, 2-16.

Perrenet J.C., Bouhuijs P.A.J. \& Smits J.G. (2000). The suitability of problem-based learning for engineering education: theory and practice. Teaching in higher education, 5(3), 345-58.

Savery J.R. (2006). Overview of Problem-based Learning: Definitions and Distinctions. Interdisciplinary Journal of Problem-Based Learning, 1(1), 8-20.

Stinson J.E. y Milter, R.G. (1996). Problem-based learning in business education: Curriculum design and implementation issues. New directions For Teaching and Learning, 1996(68), 33-42.

Woods, D.R. (1994). Problem-based learning: How to gain the most from PBL. Waterdown, Ontario: D.R. Woods.

Woods D.R., Felder R.M., Rugarcia A. y Stice J.E., (2000). The Future of Engineering Education III. Developing critical skills. Chemical Engineering Education, 34(2), 108-117.

Yadav, A., Shaver, G. M., y Meckl, P. (2010). Lessons learned: Implementing the case teaching method in a mechanical engineering course. Journal of Engineering Education, 99(1), 55-69.

Yadav A., Subedi D., Lundeberg M.A. y Bunting C. (2011). Problem-based Learning: Influence on Students' Learning in an Electrical Engineering Course. Journal of Engineering Education, 100(2), 253-80. 